\title{
Semi-Analytic Technique for Solving Fractional Partial Differential Equations with Conformable Derivatives
}

\author{
Noor I. Ibrahim and Osama H. Mohammed*
}

\begin{abstract}
Department of Mathematics, College of Science, Al-Nahrain University, Baghdad, Iraq
\end{abstract}

\begin{tabular}{|c|c|}
\hline Article's Information & Abstract \\
\hline $\begin{array}{l}\text { Received: } \\
\text { 13.02.2021 } \\
\text { Accepted: } \\
\text { 06.03.2021 } \\
\text { Published: } \\
\text { 13.03.2021 }\end{array}$ & \multirow{2}{*}{$\begin{array}{l}\text { In this work, we present a semi-analytical technique to find an approximate result of the } \\
\text { conformable fractional partial differential equations (CFPDEs). The fractional order } \\
\text { derivative will be in the conformable (CFD) sense. This definition is effective and simple in } \\
\text { the solution of the fractional differential equations that have intricate solution with classical } \\
\text { fractional derivative definition like Riemann-Liouville and Caputo. Furthermore, the result } \\
\text { obtained by the proposed technique is like those in previous studies that used other types of } \\
\text { approximate methods like (Homotopy analysis method) but it has the advantage of being } \\
\text { simpler than the rest of these methods. In addition, results demonstrate obtained the } \\
\text { Precision and effectiveness of the suggested technique. }\end{array}$} \\
\hline $\begin{array}{l}\text { Keywords: } \\
\text { Conformable fractional derivative } \\
\text { Semi-analytic technique } \\
\text { Fractional order partial } \\
\text { differential equations } \\
\text { Fractional damped Burger } \\
\text { equation }\end{array}$ & \\
\hline
\end{tabular}

DOI: $10.22401 /$ ANJS.24.1.07

*Corresponding author: uhm@sc.nahrainuniv.edu.iq

\section{Introduction}

Fractional integration and fractional differentiation has opened their wings even larger to cover the dynamics of real world and new ideas are starting to be tested and implemented on real data [1]. The concept of fractional derivatives is by no means new. In fact, they are almost as old as their more familiar integer-order counterparts until recently; however, fractional derivatives have been successfully applied to problems in system biology, physics, chemistry and biochemistry hydrology. Fractional partial differential equations (FPDEs) arise in many areas of mathematics, engineering, and the physical science, which make it very important to detect active technique for solving the partial fractional differential equations. Thus, the solutions of the fractional equations become strongly accepted [2, 3, 4, 5]. There are some definitions about fractional derivative [6,7], two of them are most usually applied the definitions of the Caputo and RiemannLiouville. The (CFD) one of the new defined fractional derivative and is defined by $\mathrm{R}$. Khalil [7]. There are many related studies to the conformable fractional order derivative. For instance, Abdeljawd [8] set the basic concepts and developed the definition of the conformable fractional derivative of this interesting fractional calculus. Atangana et al. [9] view the properties of conformable derivative. Chung [11] has discussed the (CFD) and integral to explore the fractional Newtonian Mechanics. Kurt et al. [10] using homotopy analysis method to find approximate solution of the time conformable Burger's equations specified. Gökdogan et al. [12] discuss some conformable differential equations and gave the existence and uniqueness theorems of solutions. Çenesiz et al. [13] gets new exact solutions of the conformable Burger's type equations. The reader can also see the references $[14,15$, 16], which have been also focus on the numerical and approximate solutions of (CFDEs).

\section{Preliminaries}

In this part, we prepare the definitions and some fundamental properties regarding the conformable calculus [13].

Definition 2.1. Given a function $f:[0, \infty) \rightarrow \mathbb{R}$. Then the conformable fractional derivative of $f$ of order $\alpha$ is defined by:

$$
\left(T_{\alpha} f\right)(t)=\lim _{\varepsilon \rightarrow 0} \frac{f\left(t+\varepsilon t^{1-\alpha}\right)-f(t)}{\varepsilon}
$$

for all $t>0, \alpha \in(0,1]$.

Lemma 2.1. Let $\alpha \in(0,1]$ and $f, g$ be " $\alpha$-differentiable" at a point $t>0$. Then:

(1) $T_{\alpha}(a f+b g)=a\left(T_{\alpha} f\right)+b\left(T_{\alpha} g\right)$ for $\mathrm{a}, \mathrm{b} \in \mathbb{R}$.

(2) $T_{\alpha}\left(t^{\mathrm{p}}\right)=\mathrm{p} t^{\mathrm{p}-\alpha}$, for all $p \in \mathbb{R}$.

(3) $T_{\alpha}(\lambda)=0$, for all constant function $f(t)=\lambda$.

(4) $T_{\alpha}(f g)=f\left(T_{\alpha} g\right)+g\left(T_{\alpha} f\right)$.

(5) $T_{\alpha}\left(\frac{f}{g}\right)=\frac{g\left(T_{\alpha} f\right)-f\left(T_{\alpha} g\right)}{g^{2}}$. 


\section{Al-Nahrain Journal of Science}

ANJS, Vol.24 (1), March, 2021, pp. 39-44
(6) If $f(t)$ is differentiable, then $T_{\alpha}(f(t))=$ $t^{1-\alpha} \frac{d}{d t} f(t)$

Definition 2.2. A fractional integral starting from $a$ of a function $f:[\mathrm{a}, \infty) \rightarrow \mathbb{R}$ of order $\alpha \in(n, n+1], n \in \mathbb{N}$ is defined by:

where:

$$
I_{a}^{\alpha} f(t)=\frac{1}{(n-1) !} \int_{a}^{t} \frac{(t-x)^{n-1}}{(x-a)^{n-\alpha}} d x, \alpha>0, t>a
$$

$$
I_{a}^{0} f(x)=f(x)
$$

Remark 2.1. If $\alpha \in(n, n+1], \lambda$ is a constant and $\alpha+\gamma-n>0$, then:

(1) $I_{a}^{\alpha}(t-a)^{\gamma}=\frac{\Gamma(\gamma+\alpha-n)}{\Gamma(1+\gamma+\alpha)}(t-a)^{\gamma+\alpha}$.

(2) $I_{a}^{\alpha}(\lambda)^{\gamma}=\lambda \frac{\Gamma(\alpha-n)}{\Gamma(1+\alpha)}(t-a)^{\alpha}$.

Remark 2.2. Let $\alpha \in(n, n+1]$ and $f:[a, \infty) \rightarrow \mathbb{R}$ be $(n+1)$ times differentiable for $t>a$; then we have :

$$
I_{\alpha}^{a} T_{\alpha}^{a}(f)(t)=f(t)-\sum_{k=0}^{n} \frac{f^{(k)}(a)(t-a)^{k}}{k !}
$$

\section{The Proposed Method}

In this section we will propose a semi-analytic iterative method for solving the nonlinear (CFPDEs), and to illustrate this, we need to consider the (CFPDEs)

$$
L[u(x, t)]+M[u(x, t)]+H(x, t)=0
$$
such that:

$$
u(x, 0)=b(x)
$$

where

$$
L[u(x, t)]={ }_{t} T_{\alpha}[u(x, t)]
$$

${ }_{t} T_{\alpha}$ represent the conformable derivative of the function $u$ with respect to $t$.

$M$ is a non-linear operator.

$H$ is a nonhomogeneous term.

The following steps illustrate the proposed algorithm briefly.

Step 1. We assume that $u_{0}(x, t)$ is the initial guess solution of problem (1)-(2) which can be obtained by solving the following initial value problem (IVP)

$$
L\left[u_{0}(x, t)\right]+H(x, t)=0, u_{0}(x, 0)=b(x)
$$

Step 2. To find the next iteration, we need to resolve the following linear problem:

$$
\begin{aligned}
& L\left[u_{1}(x, t)\right]+M\left[u_{0}(x, t)\right]+H(x, t)=0 \\
& u_{1}(x, 0)=b(x)
\end{aligned}
$$

Step 3. For finding the rest solutions $u_{2}(x, t), u_{3}(x, t), \ldots$; we need to solve the following linear problems

$$
\begin{aligned}
& L\left[u_{k+1}(x, t)\right]+M\left[u_{k}(x, t)\right]+H(x, t)=0 \\
& u_{k+1}(x, 0)=b(x)
\end{aligned}
$$

for all $k=1,2, \ldots$.

It is remarkable that each function $u_{0}(x, t), u_{1}(x, t)$, $u_{2}(x, t), \ldots$ can be considered as a solution to the problem (1) $-(2)$.

\section{Error Analysis}

In this part, we will discuss the error analysis of the proposed technique and for this purpose we present the consecutive errors:

$$
\begin{aligned}
\mathrm{E}_{k} & =\left\|u_{k}-u_{k-1}\right\|_{b, \lambda} \\
& =\max _{(x, t) \epsilon[0,1] \times[0,1]}\left|u_{k}(x, t)-u_{k-1}(x, t)\right| e^{-\lambda x t}
\end{aligned}
$$

which are the difference between two consecutive iterate solutions.

\section{Numerical Applications}

In this part, we will provide illustrative examples in order to give an explanation of the effectiveness and applicability of the suggested method.

Example 5.1. Consider the following fractional damped Burger equation:

$$
{ }_{t} T_{\alpha} u+u u_{x}+u_{x x}+\frac{1}{5} u=0
$$

subject to the initial condition:

$$
u(x, 0)=\frac{x}{5}
$$

when $\alpha=1$, the exact solution of problem (3)-(4) is given by [15]:

$$
u(x, t)=\frac{x}{5\left(2 e^{\frac{1}{5} t}-1\right)}
$$

Step 1. To find $u_{0}(x, t)$, the following (IVP) problem must be solved:

$$
{ }_{t} T_{\alpha}\left[u_{0}\right]=0
$$

subject to:

$$
u_{0}(x, 0)=\frac{x}{5}
$$

Integrating both sides of equation (5), yields:

$$
u_{0}(x, t)=\frac{x}{5}
$$

Step 2. $u_{1}(x, t)$, can be gained by solving the following linear (IVP)

$$
{ }_{t} T_{\alpha}\left(u_{1}\right)+u_{0}\left(u_{0}\right)_{X}+\left(u_{0}\right)_{X X}+\frac{1}{5} u_{0}=0
$$

subject to:

$$
u_{1}(x, 0)=\frac{x}{5}
$$

Hence:

$$
u_{1}(x, t)=\frac{x}{5}-\frac{2 x \Gamma(\alpha) t^{\alpha}}{25 \Gamma(\alpha+1)}
$$

Step 3. $u_{2}(x, t)$ can be obtained by solving the following linear problem:

$$
{ }_{t} T_{\alpha}\left(u_{2}\right)+u_{1}\left(u_{1}\right)_{X}+\left(u_{1}\right)_{X X}+\frac{1}{5} u_{1}=0
$$

subject to:

$$
u_{2}(x, 0)=\frac{x}{5}
$$

Therefore :

$$
\begin{aligned}
u_{2}(x, t)= & \frac{x}{5}-\frac{2 x \Gamma(\alpha) t^{\alpha}}{25 \Gamma(\alpha+1)}+\frac{6 x \Gamma(\alpha) \Gamma(2 \alpha) t^{2 \alpha}}{125 \Gamma(\alpha+1) \Gamma(2 \alpha+1)}- \\
& \frac{4 x(\Gamma(\alpha))^{2} \Gamma(3 \alpha) t^{3 \alpha}}{625(\Gamma(\alpha+1))^{2} \Gamma(3 \alpha+1)}
\end{aligned}
$$

Figures 1, 2 and 3 represent a comparison among the solutions of problem (3)-(4) that have been obtained by the suggested method with the exact solution and the existing methods when $\alpha=1, \alpha=0.9$ and 0.8 , respectively. 


\section{Al-Nahrain Journal of Science}

ANJS, Vol.24 (1), March, 2021, pp. 39-44

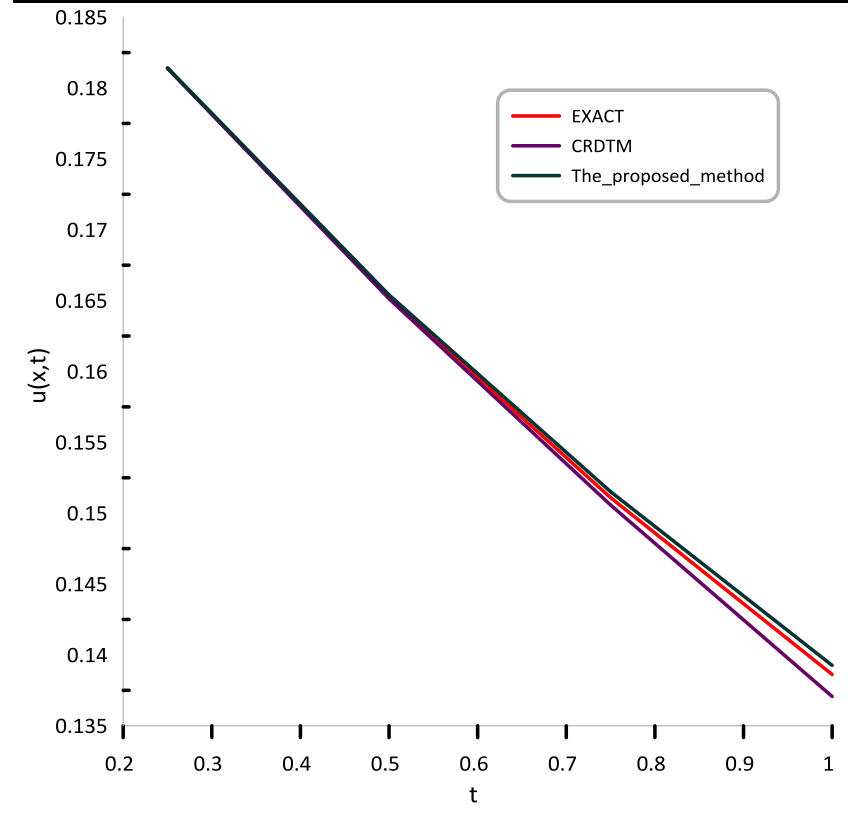

Figure 1. Results of problem (3)-(4), when $\alpha=1$.

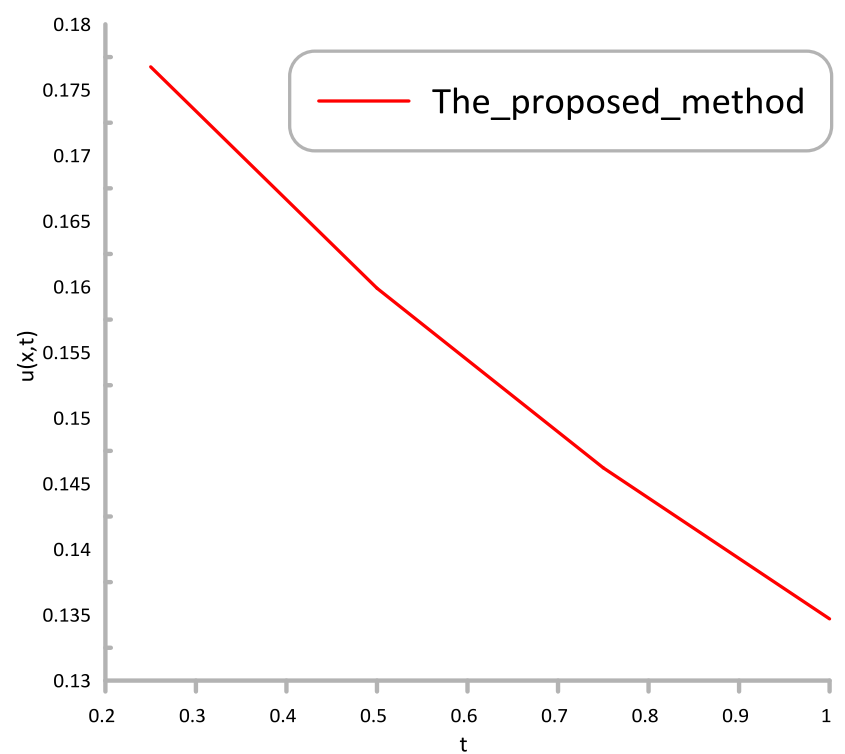

Figure 2. Results of problem (3)-(4), when $\alpha=0.9$.

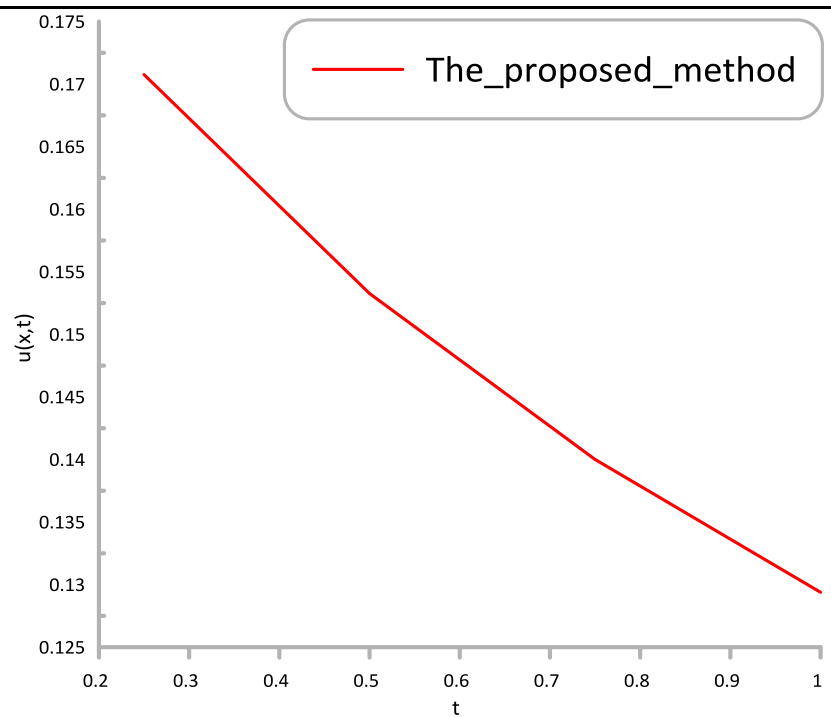

Figure 3. Results of problem (3)-(4), when $\alpha=0.8$.

Following Tables 1, 2 and 3 represent the consecutive errors of the solution of problem (3)-(4) when $\alpha=1,0.9$ and 0.8 , respectively.

Table 1. The consecutive errors of the solutions of problem (3)-(4), when $\alpha=1$.

\begin{tabular}{|c|c|}
\hline $\boldsymbol{k}$ & $\boldsymbol{E}_{\boldsymbol{k}}$ \\
\hline \hline 1 & 0.0294304 \\
\hline 2 & $8.0442971 \times 10^{-3}$ \\
\hline 3 & $4.2994255 \times 10^{-3}$ \\
\hline 4 & $3.3412054 \times 10^{-3}$ \\
\hline
\end{tabular}

Table 2. The consecutive errors of the solutions of problem (3)-(4), when $\alpha=0.9$.

\begin{tabular}{|c|c|}
\hline $\boldsymbol{k}$ & $\boldsymbol{E}_{\boldsymbol{k}}$ \\
\hline \hline 1 & 0.0327004 \\
\hline \hline 2 & $9.8235754 \times 10^{-3}$ \\
\hline 3 & $5.4409116 \times 10^{-3}$ \\
\hline 4 & $4.2953688 \times 10^{-3}$ \\
\hline
\end{tabular}

Table 3. The consecutive errors of the solutions of problem (3)-(4) when $\alpha=0.8$.

\begin{tabular}{|c|c|}
\hline $\boldsymbol{k}$ & $\boldsymbol{E}_{\boldsymbol{k}}$ \\
\hline \hline 1 & 0.0367879 \\
\hline 2 & 0.0122626 \\
\hline 3 & $7.0809037 \times 10^{-3}$ \\
\hline 4 & $5.6286944 \times 10^{-3}$ \\
\hline
\end{tabular}




\section{Al-Nahrain Journal of Science}

ANJS, Vol.24 (1), March, 2021, pp. 39-44

Example 5.2. Consider the following FPDE:

${ }_{t} T_{\alpha} u-u_{x} u_{x x}-u_{x x}+u u_{x x x}=0$

subject to the initial-condition:

$$
u(x, 0)=\frac{1}{4} e^{\frac{1}{4} x}
$$

when $\alpha=1$, the exact solution of problem (8)-(9) is given by $[15]^{\prime \prime}$

$$
u(x, t)=\frac{1}{4} e^{\frac{1}{4}\left(x+\frac{1}{4} t\right)}
$$

Step 1. To find $u_{0}(x, t)$, the following (IVP) problem must be solved

$$
{ }_{t} T_{\alpha}\left[u_{0}\right]=0
$$

subject to:

$$
u_{0}(x, 0)=\frac{1}{4} e^{\frac{1}{4} x}
$$

Integrating both sides of equation (10), yields:

$$
u_{0}(x, t)=\frac{1}{4} e^{\frac{1}{4} x}
$$

Step 2. $u_{1}(x, t)$, can be obtained by solving the following linear (IVP)

$$
{ }_{t} T_{\alpha} u_{1}-\left(u_{0}\right)_{x}\left(u_{0}\right)_{x x}-\left(u_{0}\right)_{x x}+u_{0}\left(u_{0}\right)_{x x x}=0
$$

such that:

$$
u_{1}(x, 0)=\frac{1}{4} e^{\frac{1}{4} x}
$$

Hence:

$$
\mathrm{u}_{1}(\mathrm{x}, \mathrm{t})=\frac{1}{4} \mathrm{e}^{\frac{1}{4} \mathrm{x}}+\frac{1}{64} \mathrm{e}^{\frac{1}{4} \mathrm{x}} \frac{\Gamma(\alpha) \mathrm{t}^{\alpha}}{\Gamma(\alpha+1)}
$$

Step 3. To find $u_{2}$ we must be solve the linear problem:

$$
{ }_{t} T_{\alpha} u_{2}-\left(u_{1}\right)_{x}\left(u_{1}\right)_{x x}-\left(u_{1}\right)_{x x}+u_{1}\left(u_{1}\right)_{x x x}=0
$$

such that:

$$
u_{2}(x, 0)=\frac{1}{4} e^{\frac{1}{4} x}
$$

Therefore:

$$
\begin{aligned}
u_{2}(x, t)= & \frac{1}{4} e^{\frac{1}{4} x}+\frac{1}{64} e^{\frac{1}{4} x} \frac{\Gamma(\alpha) \cdot\left(t^{\alpha}\right)}{\Gamma(\alpha+1)}+ \\
& \frac{1}{1024} e^{\frac{1}{4} x} \frac{\Gamma(\alpha) \cdot \Gamma(2 \alpha) \cdot\left(t^{2 \alpha}\right)}{\Gamma(\alpha+1) \cdot \Gamma(2 \alpha+1)}
\end{aligned}
$$

Figures 4, 5 and 6 represent a comparison among the solutions of problem (8)-(9) that have been gained by the suggested method with the exact solution and the existing methods when $\alpha=1, \alpha=0.9$ and 0.8 , respectively.

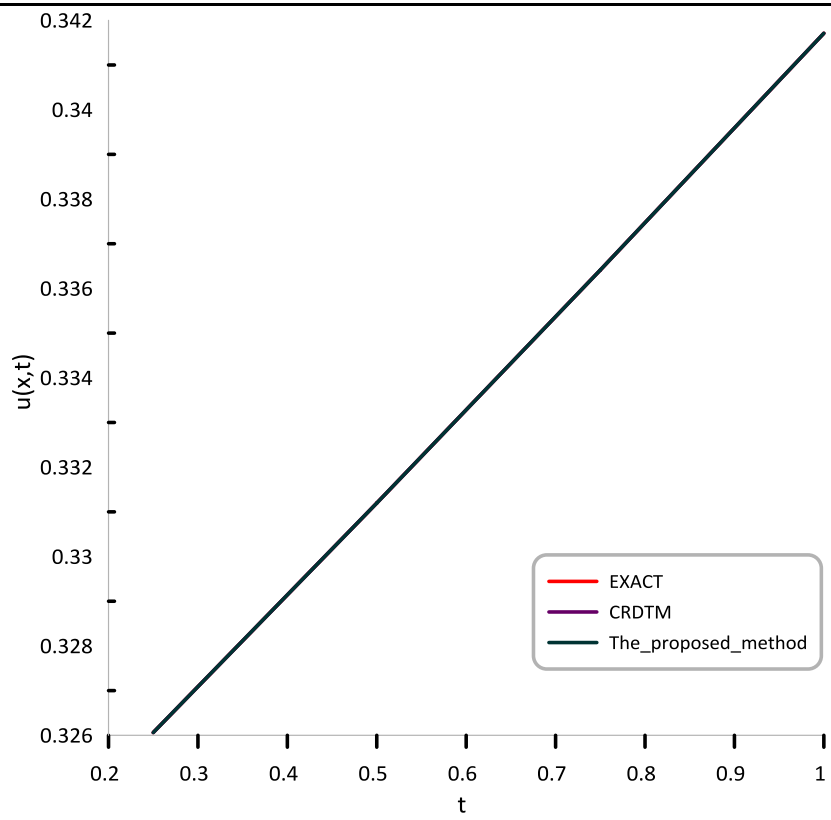

Figure 4. Results of problem (8)-(9), when $\alpha=1$.

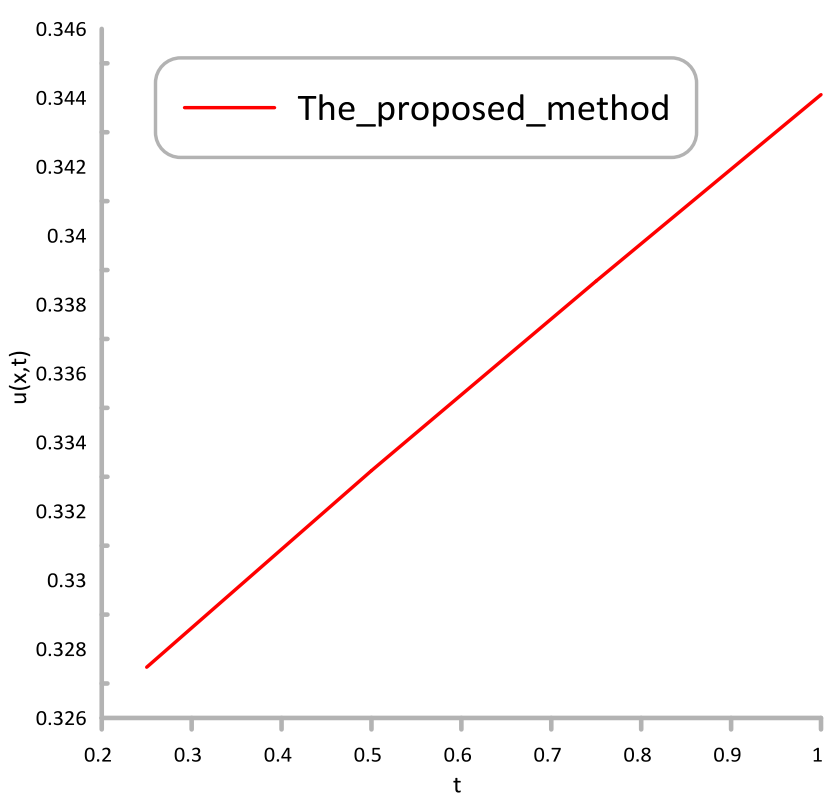

Figure 5. Results of problem (8)-(9), when $\alpha=0.9$. 


\section{Al-Nahrain Journal of Science}

ANJS, Vol.24 (1), March, 2021, pp. 39-44

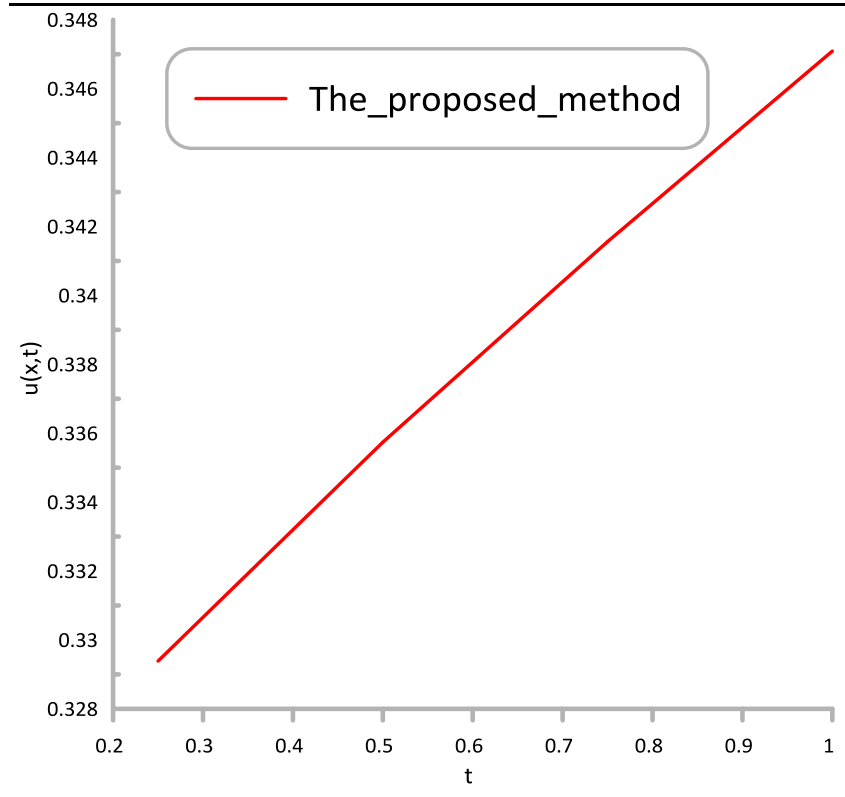

Figure 6. Results of problem (8)-(9), when $\alpha=0.8$.

Following Tables 4, 5 and 6 represent the consecutive errors of the solution of problem (8)-(9) when $\alpha=1,0.9$ and 0.8 , respectively.

Table 4. The consecutive errors of the solutions of problem (8)-(9), when $\alpha=1$.

\begin{tabular}{|c|c||}
\hline $\boldsymbol{k}$ & $\boldsymbol{E}_{\boldsymbol{k}}$ \\
\hline \hline 1 & $7.38072738 \times 10^{-3}$ \\
\hline 2 & $2.3064773 \times 10^{-4}$ \\
\hline 3 & $4.80516106 \times 10^{-6}$ \\
\hline 4 & $7.50806415 \times 10^{-8}$ \\
\hline
\end{tabular}

Table 5. The consecutive errors of the solutions of problem (8)-(9), when $\alpha=0.9$.

\begin{tabular}{|c|c||}
\hline $\boldsymbol{k}$ & $\boldsymbol{E}_{\boldsymbol{k}}$ \\
\hline \hline 1 & $8.200808207 \times 10^{-3}$ \\
\hline 2 & $2.8475028 \times 10^{-4}$ \\
\hline 3 & $6.59144178 \times 10^{-6}$ \\
\hline 4 & $1.14434753 \times 10^{-8}$ \\
\hline
\end{tabular}

Table 6. The consecutive errors of the solutions of problem (8)-(9), when $\alpha=0.8$.

\begin{tabular}{|c|c|}
\hline $\boldsymbol{k}$ & $\boldsymbol{E}_{\boldsymbol{k}}$ \\
\hline \hline 1 & $9.225909233 \times 10^{-3}$ \\
\hline 2 & $3.6038708 \times 10^{-4}$ \\
\hline 3 & $9.38508019 \times 10^{-6}$ \\
\hline 4 & $1.83302348 \times 10^{-7}$ \\
\hline
\end{tabular}

\section{Conclusions}

In this study, the conformable semi-analytic approach has been investigated. The proposed approach was applied in order to find the approximate solutions of a class of nonlinear FPDEs with conformable fractional order derivative. From the results of the illustrative examples, we have been approved that this approach is effective and accurate for solving such kind of problems.

\section{References}

[1] Sun, H. G.; Zhang, Y.; Baleanu, D.; Chen, W. and Chen Y. Q.; "A new collection of real world applications of fractional calculus in science and engineering"; Commun Nonlinear Sci Numer Simulat, 64, 213-231, 2008.

[2] Diethelm, K. and Ford J.; "Numerical solution of the bagley-torvik equation"; BIT Numer. Math., 42(3), 490-507, 2002.

[3] Zhuang; P; Liu, F. and Anh, V.; "New solution and analytical techniques of the implicit numerical method for the anomalous subdiffusion equation"; SIAM J. Numer. Anal., 46(2), 1079-1095, 2008.

[4] Liu, F.; Yang, C. and Burrage, K.; "Numerical method and analytical technique of the modified anomalous subdiffusion equation with a nonlinear source term"; Comput Appl. Math., 231(1), 160-176, 2009.

[5] Jiang, Y. and Ma, J.; "High-order finite element methods for time-fractional partial differential equations"; Comput Appl. Math., 235(11), 3285-3290, 2011.

[6] Podlubny, I.; "Fractional differential equations"; Academic Press, New York, 1999.

[7] Khalil, R.; Al Horani, M. and Yousef, A.; "A new definition of fractional derivative"; Comput Appl. Math., 264, 65-70, 2014.

[8] Abdeljawad, T.; "On conformable fractional calculus"; Comput. Appl. Math., 279, 57-66, 2015.

[9] Atangana, A.; Baleanu, D. and Alsaedi, A.; "New properties of conformable derivative"; Open Math., 13(1), 889-898, 2015.

[10] Kurt, A.; Enesiz, Y. and Tasbozan, O.; "On the solution of Burgers' equation with the new fractional derivative"; Open Phys., 13(1), 355-360, 2015.

[11] Chung, W. S.; "Fractional newton mechanics with conformable fractional derivative"; Comput Appl. Math., 290, 150-158, 2015.

[12] Gökdogan, A.; Ünal, E. and Celik, E.; "Existence and uniqueness theorems for sequential linear conformable fractional differential equations"; Miskolc Math. Notes, 267-279, 2016.

[13] Çenesiz, Y.; Baleanu, D. and Kurt, A.; "New exact solutions of Burgers' type equations with conformable derivative"; Waves Random Complex Media, 27(1), 103-116, 2017.

[14] Ünal, E. and Gökdoğan, A.; "Solution of conformable fractional ordinary differential equations via 


\section{Al-Nahrain Journal of Science}

ANJS, Vol.24 (1), March, 2021, pp. 39-44

differential transform method"; Light Electron, 12, 264-273, 2017.

[15] Acan, O.; Firat, O. and Keskin, Y.; "Conformable variational iteration method, conformable fractional reduced differential transform method and conformable homotopy analysis method for nonlinear fractional partial differential equations"; Waves in Random and Complex Media, 2018.

[16] Malik, A. M. and Mohammed, O. H.; "Two efficient methods for solving fractional Lane Emden equations with conformable fractional derivative"; Egyptian Mathematical Society, 28(42), 2020. 